Research Article

\title{
A New Blind Source Separation Algorithm Framework for Noisy Mixing Model Based on the Energy Concentration Characteristic in Signal Transform Domain
}

\author{
Jiong Li iD and Lu Feng \\ Space Engineering University, Beijing 101416, China \\ Correspondence should be addressed to Jiong Li; lij_2015@126.com
}

Received 2 August 2021; Revised 26 September 2021; Accepted 9 December 2021; Published 29 December 2021

Academic Editor: Ricardo Escobar

Copyright (c) 2021 Jiong Li and Lu Feng. This is an open access article distributed under the Creative Commons Attribution License, which permits unrestricted use, distribution, and reproduction in any medium, provided the original work is properly cited.

\begin{abstract}
Blind source separation is a widely used technique to analyze multichannel data. In most real-world applications, noise is inevitable and will affect the quality of signal separation and even make signal separation failure. In this paper, a new signal processing framework is proposed to separate noisy mixing sources. It is composed of two different stages. The first step is to process the mixing signal by a certain signal transform method to make the expected signals have energy concentration characteristics in the transform domain. The second stage is formed by a certain BSS algorithm estimating the demixing matrix in the transform domain. In the energy concentration segment, the SNR can reach a high level so that the demixing matrix can be estimated accurately. The analysis process of the proposed algorithm framework is analyzed by taking the wavelet transform as an example. Numerical experiments demonstrate the efficiency of the proposed algorithm to estimate the mixing matrix in comparison with well-known algorithms.
\end{abstract}

\section{Introduction}

Blind source separation (BSS) is a research hotspot in the field of signal processing in recent years, which can estimate source signals from the mixed signals with little information of source signals and mixing system parameters. Since the power and frequency characteristics of the signal is not limited in BSS, it has attracted many experts and scholars in many research fields, and it has been wildly used in speech signal processing, image signal processing, communication signal processing, and medical signal processing.

According to the relationship between the number of source signals and the number of receiving sensors, the signal mixing model can be divided into three types, namely, under-determined mixing (the number of source signals is more than the number of receiving sensors), well-determined mixing (the number of source signals is equal to the number of receiving sensors), and over-determined mixing (the number of source signals is lower than the number of receiving sensors). The signal mixing model also can be divided into linear instantaneous mixing and convolutional mixing according to the transmission response characteristics of the mixing channel. Regardless of the mixing model, the current BSS theory is mostly based on independent component analysis (ICA) [1]. Comon [1] gives three assumptions for using the ICA method to realize BSS. One is that the source signals are independent of each other. Second, there is at most one Gaussian signal in the source signals. Third, the channel transmission matrix is full rank. Comon [1] also summarizes the method of studying BSS based on ICA, that is, constructing a cost function based on certain criteria and obtaining the separation parameters by solving the maximum (minimum) of the cost function so that the correlation of each source signal in the mixed signal is minimized, and then, the source signals are estimated from the mixed signal. Based on the proposal of this idea, for different application scenarios, combined with the prior information of the signal (such as limited character set 
characteristics, time structure characteristics, and nonstationary characteristics), a large number of scholars have emerged to engage in related research and published a large number of literature. Many new technologies are introduced into BSS research. For example, signal sparse component analysis $[2,3]$, dictionary learning $[4,5]$, nonnegative matrix factorization $[6,7]$, bounded component analysis $[8,9]$, tensor decomposition [10, 11], and machine learning [12]. However, these algorithms are sensitive to noise. In practical applications, noise is inevitable, even very large. For example, spread spectrum communication system works at very low signal-to-noise ratio (SNR). Therefore, in order to make the BSS technology practical, it is necessary to consider the problem of blind source separation in the presence of noise.

There are two research ideas about BSS in the presence of noise. One is to first use denoise technology to eliminate or suppress the noise in the received signal to improve the SNR of the received signal, and then, the sources are estimated from the denoised signals by BSS algorithm. In this way, noise suppression and BSS are two independent modules, so it is difficult to fundamentally improve the robustness of the separation results. The other is to consider the influence of noise when designing the BSS algorithm so as to improve the antinoise robustness of the BSS algorithm. There are mainly two ways to implement it. One is to use the characteristic that the high-order cumulant of Gaussian distribution is zero to deal with the blind separation problem with noise. However, since high-order cumulants are sensitive to singular values, so this type of method has a low robustness. The second implementation method is to analyze the received signal using a probability graph model and then use Bayesian inference to estimate the model parameters and the source signals. Noise is considered in the analysis of the received signal probability graph model. Generally speaking, there are so many parameters to be estimated that the algorithms are very complex. In addition, the performance of these algorithms is sensitive to initial parameters, and the estimated parameters are prone to overfitting.

Considering that the source signals are mostly energydispersed time-domain signals, the energy can be concentrated by some special signal transform methods. This paper proposes a blind separation algorithm framework based on signal transform. The algorithms use the energy concentration characteristics of the signal transform and select the signal segments with concentrated energy as the analysis object. The SNR of the signal segments reaches a high level, and the demixing matrix can be estimated to separate the source signals. This method can effectively reduce the influence of singular values on the separation results, and the model parameters are small and almost no overfitting.

The remainder of this paper is organized as follows. Section 2introduces the system model used in this paper. Next, the proposed algorithm framework is described in Section 3. An example of the proposed BSS method is introduced in Section 4. Section 5demonstrates our experimental results, and conclusions are drawn in Section 6.

\section{System Model}

Assuming that there are Nsource signals $\left\{s_{j}(t), j=\right.$ $1,2, \ldots, N\}$ and $M$ receiving sensors, the signal received by the $i$ th receiving sensor is $r_{i}(t), i=1,2, \ldots, M$. The mixing system model is shown in Figure 1.

The mathematical model of the mixing system can be expressed as

$$
R(t)=H S(t)+n(t)
$$

where $S(t)=\left[s_{1}(t) s_{2}(t) \cdots s_{N}(t)\right]^{T}(t=0,1,2, \ldots, L-1)$, $H$ is the transmission matrix with $M \times N$, and the element $h_{i j}(i=1,2, \ldots, M ; j=1,2, \ldots, N)$ of $H$ represents the transmission attenuation coefficient from the $j$ th source signal to the $i$ th sensor. Generally, it is easy to set more sensors than source signals $(M>N)$, and this over-determined mixing model can be transformed to well-determined mixing model $(M=N)$ by using principle component analysis (PCA). So, we focus on the well-determined mixing model $(M=N)$ in this paper. $n(t)=\left[n_{1}(t) n_{2}\right.$ $\left.(t) \cdots n_{M}(t)\right]^{T}$ is the noise vector existing in the system and is independent of the source signal vector $S(t) . R(t)=$ $\left[r_{1}(t) r_{2}(t) \cdots r_{N}(t)\right]^{T}$ is the receiving signal vector. Each received signal $r_{i}(t)$ contains Nsource signals, and these source signals interfere with each other; then, it is difficult for the sensors to demodulate the received signals correctly. Therefore, an interference suppressor is necessary before the received signals are demodulated. Generally, notch filtering or beamforming methods are used to suppress interference. However, when the source signals are working at the same frequency and are all in the receiver's main lobe band, these interference suppression methods cannot work normally. BSS has the ability to separate source signals from their mixed signals without limiting the frequency and power characteristics of source signals. Therefore, blind source separation technology can effectively solve the above problem. The main work of BSS is to design a demixing system $B$ at the receiver so that, after the received signal $R(t)$ is processed by the system, the output signal $\widehat{S}(t)=B R(t)$ is an effective estimation of the source signal $S(t)$, where $B$ is also called the demixing matrix. The traditional BSS method mainly focuses on the ideal mixing model where noise does not present, and they are sensitive to noise. However, channel noise is inevitable in wireless communication transmission. Even some special communication systems such as satellite communication may work under extremely low SNR conditions.

\section{Proposed Algorithm Framework}

Generally, a set of transform bases $\left\{\varphi_{i}(t), i=0,1,2, \ldots\right\}$ is chosen for signal transform firstly, which can be orthogonal or nonorthogonal. Then, the transformed signal is obtained by calculating the projection of the signal on this group of bases:

$$
T[s(t)]=\int_{-\infty}^{\infty} s(t) \varphi(t) \mathrm{d} t
$$




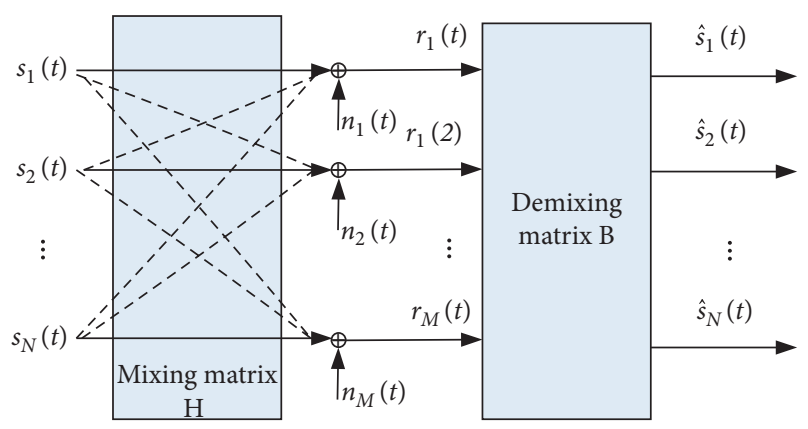

Figure 1: The noisy mixing system model.

This type of the signal transform method usually has linear characteristic and can be expressed as follows. If $s_{1}(t) \longleftrightarrow T\left[s_{1}(t)\right]$ and $s_{2}(t) \longleftrightarrow T\left[s_{2}(t)\right]$, then

$$
k_{1} s_{1}(t)+k_{2} s_{2}(t) \longleftrightarrow k_{1} T\left[s_{1}(t)\right]+k_{2} T\left[s_{2}(t)\right] .
$$

From the above signal transform on the received signal in the mixed model 1 ,we can obtain

$$
\begin{aligned}
T[R(t)] & =T[H S(t)]+T[n(t)] \\
& =H T[S(t)]+T[n(t)] .
\end{aligned}
$$

It can be concluded from (1) and (4) that the signal mixing model remains unchanged between time domain and transform domain. Therefore, the demixing matrix of (4) is also the demixing matrix of (1). In other words, we only need to calculate the demixing matrix of (4) to get the demixing matrix of (1). For some special transform methods, the signal with energy dispersion in the time domain has energy concentration characteristics in the transform domain, such as wavelet transform and discrete cosine transform (DCT) transform. The SNR of energy concentration signal segment in transform domain is higher than that of time-domain signal so that a better demixing matrix can be estimated from the signal in transform domain. The signal processing flow diagram is shown in Figure 2.

\section{An Example of Proposed Algorithm Based on Wavelet Transform}

Assuming received signal is $f(t)=s(t)+n(t)$, where $s(t)$ is expect signal and $n(t)$ is additive white Gaussian noise. The discrete wavelet transform of $f(t)$ is

$$
W(j, k)=2^{-j / 2} \sum_{m=0}^{L-1} f(m) \psi\left(2^{-j} m-k\right),
$$

where $\psi(x)$ is the wavelet basis, jis scaling factor, $k$ is translation factor, and $W(j, k)$ is wavelet coefficient. In practical applications, the wavelet transform is usually implemented by the recursive method of two-scale transform:

$$
\left\{\begin{array}{l}
W^{l}(j+1, k)=W(j, k) * l(j, k), \\
W^{h}(j+1, k)=W(j, k) * h(j, k),
\end{array}\right.
$$

where $l(\cdot)$ and $h(\cdot)$ are the low-pass and high-pass filters corresponding to the wavelet basis $\psi(x)$, respectively, $W^{l}(j, k)$ is the approximate coefficient, and $W^{h}(j, k)$ is the detail coefficient. $W^{l}(j-1, k)$ can be reconstructed by

$$
W^{l}(j-1, k)=W^{l}(j, k) * \widehat{l}(j, k)+W^{h}(j, k) * \widehat{h}(j, k),
$$

where $\widehat{l}(\cdot)$ and $\widehat{h}(\cdot)$ are the conjugate transpose of $l(\cdot)$ and $h(\cdot)$, respectively, and $W^{l}(0, k)$ is the wavelet representation of $f(m)$. If $J$-layer two-scale wavelet decomposition is performed on the signal, then the signal energy is mainly distributed on $W^{l}(J-1, k)$. Figure 3 is a schematic diagram of the three-layer wavelet decomposition of $f(m)$.

In the three-layer wavelet decomposition, the noise energy is mainly distributed on $W^{h}(1, k)$ and $W^{h}(2, k)$, and the signal energy is mainly distributed on $W^{l}(2, k)$. Combining (1), (5), and (6), we can obtain

$$
\begin{aligned}
W_{R}^{l}(j, k) & =W_{R}(j-1, k) * l(j-1, k) \\
& =2^{-(j-1)} \sum_{m=0}^{L-1} R(m) \psi\left(2^{-(j-1)} m-k\right) * l(j-1, k) \\
& =2^{-(j-1)} \sum_{m=0}^{L-1}[H S(m)+n(m)] \psi\left(2^{-(j-1)} m-k\right) * l(j-1, k) \\
& =H W_{s}^{l}(j, k)+W_{n}^{l}(j, k), \\
W_{R}^{h}(j, k)=W_{R}(j-1, k) * h(j-1, k) & =2^{-(j-1) / 2} \sum_{m=0}^{L-1} R(m) \psi\left(2^{-(j-1)} m-k\right) * h(j-1, k)
\end{aligned}
$$




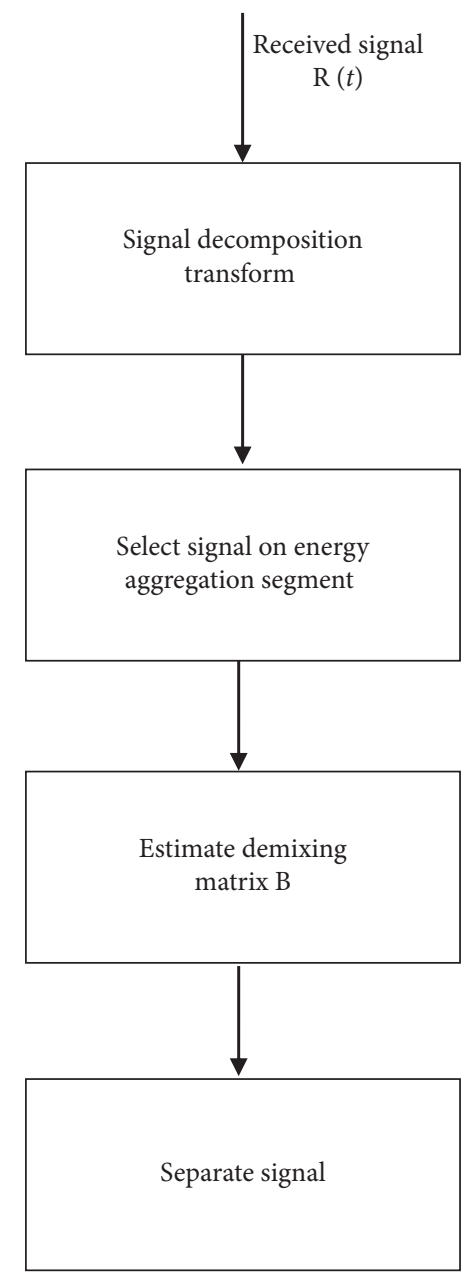

FIGURE 2: Flowchart of signal separation algorithm based on signal transform.

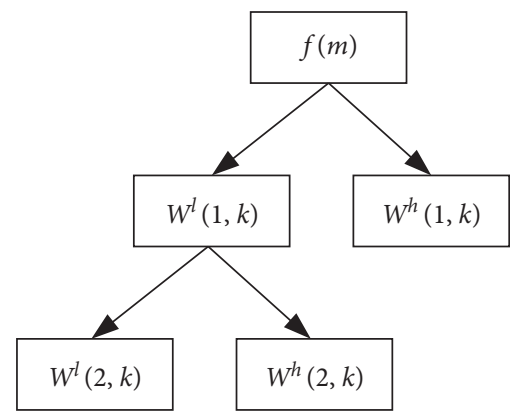

FIGURE 3: Schematic diagram of three-layer wavelet decomposition.

$$
\begin{aligned}
& =2^{-(j-1) / 2} \sum_{m=0}^{L-1}[H S(m)+n(m)] \psi\left(2^{-(j-1)} m-k\right) * h(j-1, k) \\
& =H W_{s}^{h}(j, k)+W_{n}^{h}(j, k), \\
\text { where } W_{s}^{l}(j, k) & =2^{-(j-1) / 2} \sum_{m=0}^{L-1} S(m) \psi\left(2^{-(j-1)} m-k\right) * l(j-1, k),
\end{aligned}
$$




$$
\begin{aligned}
& W_{n}^{l}(j, k)=2^{-(j-1) / 2} \sum_{m=0}^{L-1} n(m) \psi\left(2^{-(j-1)} m-k\right) * l(j-1, k), \\
& W_{s}^{h}(j, k)=2^{-(j-1) / 2} \sum_{m=0}^{L-1} S(m) \psi\left(2^{-(j-1)} m-k\right) * h(j-1, k), \\
& W_{n}^{h}(j, k)=2^{-(j-1 / 2)} \sum_{m=0}^{L-1} n(m) \psi\left(2^{-(j-1)} m-k\right) * h(j-1, k) .
\end{aligned}
$$

The signal models of (1), (4), and (8) are similar, and they have the same mixing matrix $H$, so the demixing matrix of (8) can also correctly separation the signal in (1). In addition, since noise energy is mainly distributed on $W_{R}^{h}(\cdot, k)$ and $W_{R}^{l}(\cdot, k)$ mainly carries signal energy, the SNR in (8) is higher than that in (1). Therefore, we can get a better demixing matrix from (8). The demixing of (8) is estimated by equivariant adaptive separation via independence (EASI) algorithm [13]. The objective function in EASI algorithm is the mutual information of demixed signals, and the natural gradient algorithm is used to iteratively optimize the demixing matrix. The iterative update process of the demixing matrix is as follows:

$$
\begin{aligned}
B= & B-\alpha\left\{E \left[\widehat{W}_{R}^{l}(2, k) \widehat{W}_{R}^{l}(2, k)^{T}-I\right.\right. \\
& \left.\left.+g\left(\widehat{W}_{R}^{l}(2, k)\right) \widehat{W}_{R}^{l}(2, k)^{T}-\widehat{W}_{R}^{l}(2, k) g\left(\widehat{W}_{R}^{l}(2, k)\right)^{T}\right]\right\} B,
\end{aligned}
$$

where $\alpha$ is a balance factor between 0 and $1, g(\bullet)$ is a nonlinear function, examples of choices are $g(x)=x^{3}$ for sub-Gaussian sources, $g(x)=x e^{-x^{2} / 2}$ for sup-Gaussian

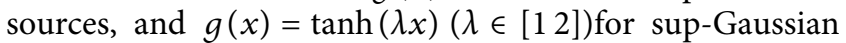
and sub-Gaussian sources.

The source signal coefficients in transform domain are estimated by $\widehat{W}_{R}^{l}(2, k)=B W_{R}^{l}(2, k)$ and $\widehat{W}_{R}^{h}(j, k)=$ $B W_{R}^{h}(j, k)$ if demixing matrix Bconverges. And then, the wavelet denoise method can be used to suppress the noise in estimated signals.

\section{Simulation Results}

First of all, the SNR is defined as the ratio of the energy of the mixed source signal to the energy of the noise in the observed signal:

$$
\operatorname{SNR}=\frac{\operatorname{Var}\left(\sum_{j=1}^{N} a_{i j} s_{j}(t)\right)}{\operatorname{Var}\left(n_{i}(t)\right)},
$$

where $\operatorname{Var}(\cdot)$ is the function to calculate the variance of $\cdot$. We use, for our performance measure, the normalized Amari index $P I \in[0,1]$ defined in $[14]$ as

$$
\begin{aligned}
P I= & \frac{1}{2 N}\left[\sum_{i=1}^{N}\left(\sum_{j=1}^{N} \frac{|G(i, j)|^{2}}{\max _{l}|G(i, l)|^{2}}-1\right)\right. \\
& \left.+\sum_{i=1}^{N}\left(\sum_{j=1}^{N} \frac{|G(j, i)|^{2}}{\max _{l}|G(l, i)|^{2}}-1\right)\right],
\end{aligned}
$$

where $G(i, j)$ is the $(i, j)$ thentry of matrix $G=B H$. The lower the PIvalue is, the better the separation performance becomes. PIalso can be regarded as the average interferenceto-signal ratio of the separated signal.

In the simulations, two BPSK modulation signals are chosen as expected signal and interference signal, respectively, and they have the same modulation parameters. The shaping filter of BPSK is root raised cosine roll-off filter, rolloff factor is 0.35 , and symbol rate is $128 \mathrm{kbps}$. The signal sampling rate is $f_{s}=1 \mathrm{MHz}$. The mixing matrix Ais generated randomly, and the elements of Aare independent of each other and obey Gaussian distribution. Each simulation result is the average value obtained from 10000 Monte Carlo simulations. Since BPSK modulation signal is sub-Gaussian, $g(x)=x^{3}$ is chosen in the simulations.

Figure 4shows the signal separation accuracy performance curve of the algorithm proposed in this paper under different SNR. There are two comparison algorithms in this simulation. One is traditional ICA algorithm. In another comparison algorithm, the received signals are denoised by wavelet denoise algorithm, and then, source signals are estimated by traditional ICA. It can be seen from Figure 4that when $S N R=5 \mathrm{~d} B$, the performance of the algorithm proposed in this paper can reach $P I=-15 \mathrm{~d} B$, while the two comparison algorithms can achieve $P I=-15 \mathrm{~d} B$ at $S N R=12.5 \mathrm{~d} B$ and $10 \mathrm{~d} B$, respectively. In the case of the same separation performance, that is, the interference-to-signal ratio of the separated signals is the same; the SNR required by the proposed algorithm is much lower than that of the two comparison algorithms.

Figure 5shows the standard deviation of the PI estimated in Figure 4with different SNR. The smaller the standard deviation of the PI, the better the antinoise robustness of the algorithm. From Figure 5, we observe that, with the increase of SNR, the standard deviation of PI decreases. The antinoise 


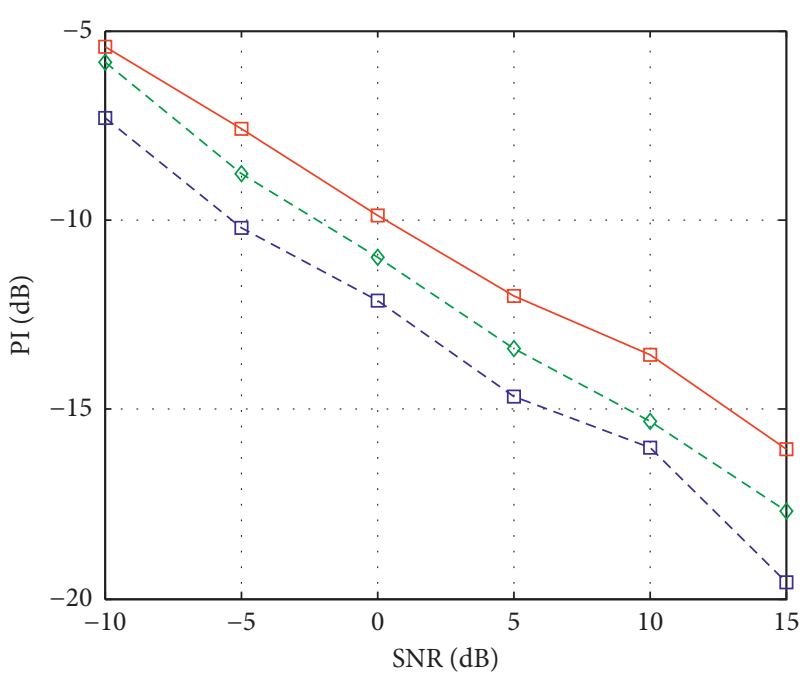

-曰- proposed algorithm

$\square$ ICA

$-\diamond-$ wavelet denoise+ICA

FIgURE 4: Separation accuracy performance curve with different SNR. The data length $L=13000$.

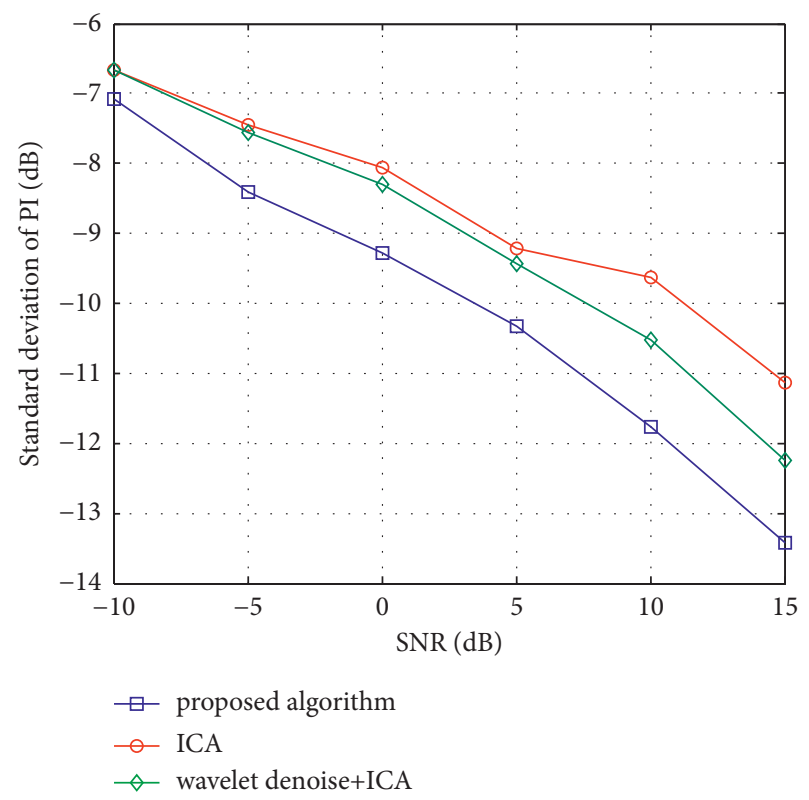

FIGURE 5: Standard deviation of estimated PI with different SNR. The data length $L=13000$.

robustness of the proposed algorithm is much better than that of the two comparison algorithms.

Figures 6and 7show the averaged PI and its standard deviation with different sample length. From the two figures, we observe that, with the increase of sample length, the PI and its standard deviation decrease. This shows that the performance of these algorithms improves as the sample length increases. And also, whether it is the separation accuracy of the algorithm or the robustness of the algorithm, the proposed algorithm is much better than that of the comparison algorithms.

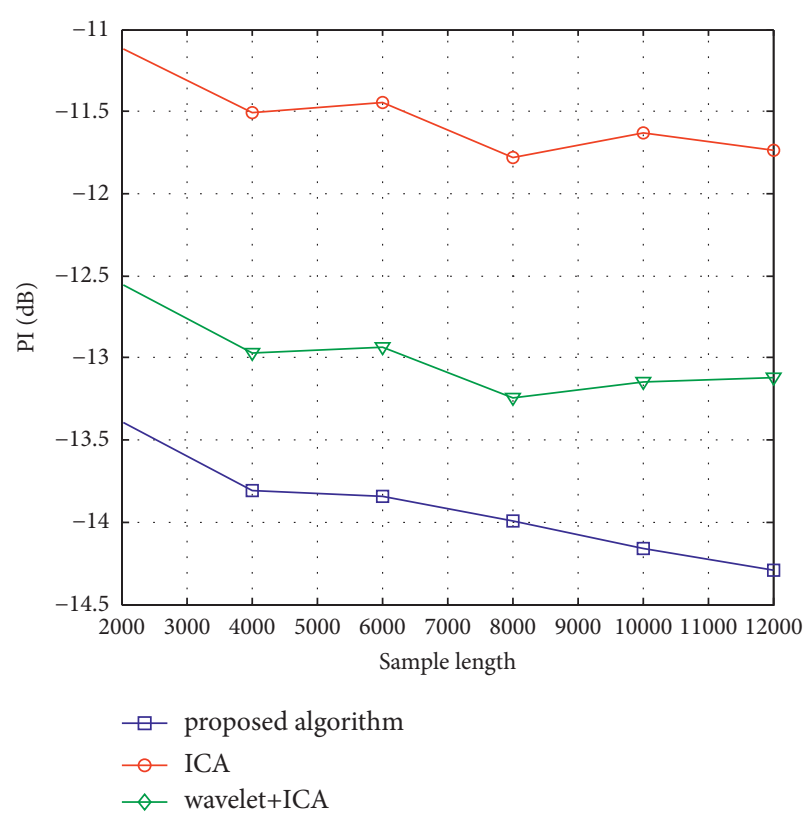

FIGURE 6: Separation accuracy performance curve with different sample lengths, $S N R=5 \mathrm{~d} B$.

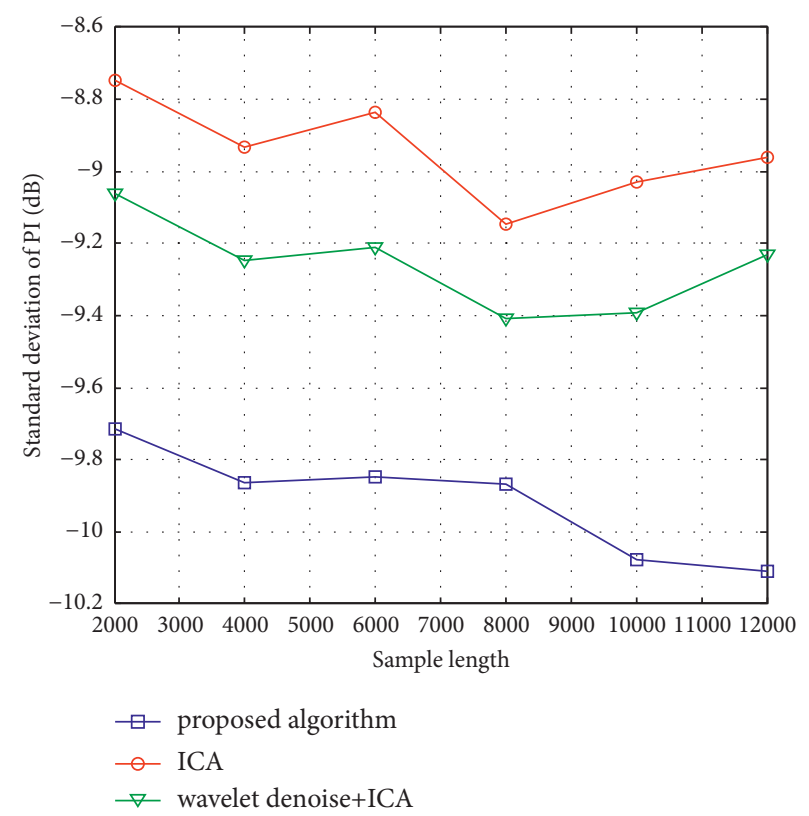

FIGURE 7: Standard deviation of estimated PI with different sample lengths, $S N R=5 \mathrm{~d} B$.

\section{Conclusion}

We introduce a new method to separate unknown sources in the presence of noise. The proposed method relies on the signal energy concentration characteristics in the transform domain. This strategy allows us to implement a two-stage scheme that makes the blind source separation algorithm work at high SNR, which is shown to highly reduce the impact of noise on the estimated sources. Numerical experiments demonstrate the good and consistent performances of the proposed algorithm to estimate the mixing 
matrix. Future work will focus on studying more signal transformation methods that match the proposed algorithm framework and how nonstationary noise affects the algorithm.

\section{Data Availability}

The data used in this study are all randomly generated using Matlab software.

\section{Conflicts of Interest}

The authors declare that they have no conflicts of interest.

\section{Acknowledgments}

This work was supported by the National Natural Science Foundation of China, under Grant 62001516.

\section{References}

[1] P. Comon, "Independent component analysis, a new concept?” Signal Processing, vol. 36, no. 3, pp. 287-314, 1994.

[2] F. Feng and M. Kowalski, "Underdetermined reverberant blind source separation: sparse approaches for multiplicative and convolutive narrowband approximation," IEEE/ACM Transactions on Audio, Speech, and Language Processing, vol. 27, no. 2, pp. 442-456, 2019.

[3] E. Babatas and A. T. Erdogan, "Sparse bounded component analysis for convolutive mixtures," in Proceedings of the presented at the 2018 IEEE International Conference on Acoustics, Speech and Signal Processing (ICASSP), Calgary, AB, Canada, April 2018.

[4] A. Dong, O. Simeone, A. M. Haimovich, and J. A. Dabin, "Blind sparse estimation of intermittent sources over unknown fading channels," IEEE Transactions on Vehicular Technology, vol. 68, no. 10, pp. 9861-9871, 2019.

[5] C. Laroche, M. Kowalski, H. Papadopoulos, and G. Richard, "Hybrid projective nonnegative matrix factorization with drum dictionaries for harmonic/percussive source separation," IEEE/ACM Transactions on Audio, Speech, and Language Processing, vol. 26, no. 9, pp. 1499-1511, 2018.

[6] J. J. Carabias-Orti, J. Nikunen, T. Virtanen, and P. VeraCandeas, "Multichannel blind sound source separation using spatial covariance model with level and time differences and nonnegative matrix factorization," IEEE/ACM Transactions on Audio, Speech, and Language Processing, vol. 26, no. 9, pp. 1512-1527, 2018.

[7] Y. Mitsufuji, S. Uhlich, N. Takamune, D. Kitamura, S. Koyama, and H. Saruwatari, "Multichannel non-negative matrix factorization using banded spatial covariance matrices in wavenumber domain," IEEE/ACM Transactions on Audio, Speech, and Language Processing, vol. 28, pp. 49-60, 2020.

[8] E. Babatas and A. T. Erdogan, "An algorithmic framework for sparse bounded component analysis," IEEE Transactions on Signal Processing, vol. 66, no. 19, pp. 5194-5205, 2018.

[9] H. A. Inan, A. T. Erdogan, and S. Cruces, "Stationary point characterization for a class of bca algorithms," IEEE Transactions on Signal Processing, vol. 65, no. 20, pp. 5437-5452, 2017.

[10] J. P. C. L. da Costa, R. K. Miranda, and M. da Rosa Zanatta, "Tensor based blind source separation for current source density analysis of evoked potentials from somatosensory cortex of mice," in Proceedings of the presented at the 2017 8th International IEEE/EMBS Conference on Neural Engineering (NER), Shanghai, China, May 2017.

[11] M. Bousse, O. Debals, and L. De Lathauwer, "A tensor-based method for large-scale blind source separation using segmentation," IEEE Transactions on Signal Processing, vol. 65, no. 2, pp. 346-358, 2017.

[12] I. T. Lee, Machine learning algorithms for independent vector analysis and blind source separation, $\mathrm{PhD}$ Thesis, University of California at San Diego, California, CA USA, 2009.

[13] J.-F. Cardoso and B. H. Laheld, "Equivariant adaptive source separation," IEEE Transactions on Signal Processing, vol. 44, no. 12 , pp. 3017-3030, 1996.

[14] S. Amari, A. Cichocki, and H. H. Yang, "A new learning algorithm for blind source separation," In Advances in Neural Information Processing Systems, vol. 8, pp. 757-763, 1996. 